\title{
Anabases
}

ANABASES Traditions et réceptions de l'Antiquité

19 | 2014

Varia

\section{La formation de Moses Finley d'après les documents américains, 1932-1955}

Daniel Tompkins

\section{OpenEdition}

1 Journals

Édition électronique

URL : http://journals.openedition.org/anabases/4622

DOI : 10.4000/anabases.4622

ISSN : 2256-9421

Éditeur

E.R.A.S.M.E.

Édition imprimée

Date de publication : 1 avril 2014

Pagination : 111-129

ISSN : 1774-4296

\section{Référence électronique}

Daniel Tompkins, «La formation de Moses Finley d'après les documents américains, 1932-1955 »,

Anabases [En ligne], 19 | 2014, mis en ligne le 01 avril 2017, consulté le 21 octobre 2019. URL : http:// journals.openedition.org/anabases/4622 ; DOI : 10.4000/anabases.4622

(c) Anabases 
Anabases 19 (2014), p. 111-129

\section{La formation de Moses Finley d'après les documents américains, 1932-1955*}

DANIEL TOMPKINS

D ANS Un très Bel article, Henri Mendras a célébré l'éclat parisien des études sur la paysannerie : "Dans ce creuset parisien, se sont noués les fils les plus divers, parce que l'extraordinaire révolution sociale et culturelle s'imposait aux yeux des sociologues et qu'elle était l'objet de toutes leurs enquêtes aux quatre coins de la France ${ }^{1}$. " Le terme " creuset " traduit aussi l'effervescence cosmopolite de New York dans les décennies qui suivirent l'arrivée de Moses Finkelstein dans cette ville en 1927. Comme le Paris de Mendras, New York fit bon accueil aux intellectuels audacieux venus des quatre coins du pays ou de l'Europe. Dans les deux villes, la socialisation intellectuelle était prépondérante, une collaboration dynamique, de celles qui continueraient à jalonner la carrière universitaire de Finley. À Paris, la guerre et l'Occupation portèrent un grand coup à cette activité, tandis que l'hostilité politique et la répression ruinèrent ou freinèrent les ambitions universitaires de presque tous les collègues de Finley, coûtant à certains leur situation pendant des décennies, ou pour toujours. Deux d'entre eux se retrouvèrent en prison ${ }^{2}$. Deux autres, Finley et Daniel Thorner, quittèrent le pays.

\footnotetext{
* La traduction de cet article a été réalisée par Franz Regnot grâce au soutien de la "Fondation Maison des Sciences de l'Homme".

1 H. MEndras, "L'invention de la paysannerie : un moment de l'histoire de la sociologie française d'après-guerre ", Revue française de sociologie 41 (2000), p. 550.

2 D. Martin, "Morris Schappes dies at 97 ; Marxist and Jewish scholar ", New York Times, 9 juin 2004 ; "Second man [Samuel Coleman] freed on bail in red case ", Los Angeles Times, 19 novembre 1954.
} 
Herbert Norman, brillant historien du Japon, se donna la mort au Caire ${ }^{3}$. Finley et Thorner apportèrent une contribution importante au débat sur la paysannerie chanté par Mendras, mais ce fut en tant qu'exilés.

Moses Finley aurait été d'accord avec Pierre Vidal-Naquet, qui voyait en Max Weber " notre père à tous ${ }^{4}$ ". Il est sûr que Weber exerça une profonde influence sur lui, Karl Polanyi un peu moins ${ }^{5}$. Mais je m'attacherai ici aux premiers contacts de Finley avec le marxisme et à ses premières activités au sein de la gauche car, chose curieuse, ces éléments très importants de sa paideia ont souvent été négligés ${ }^{6}$. Bien qu'il n'ait pas attiré l'attention sur ce point, il y a une continuité évidente lorsque, à ses débuts, il approuve Ciccotti, qui souligne que "l'homme est et a toujours été une créature sociale ", et lorsqu'il adopte plus tard les trois conditions nécessaires pour l'esclavage énoncées par Istvan Hahn : la propriété privée, la production de marchandises et l'absence de main d'œuvre interne ${ }^{7}$. Bien que l'on ait parfois attaqué son œuvre comme " antimarxiste ${ }^{8}$ ", un examen attentif montre qu'il a bien étudié l'historiographie marxiste pendant toute sa carrière et qu'il l'a parfois défendue, notamment dans Ancient Slavery and Modern Ideology. À la fin de son article sur le projet d'histoire économique de la Deutsche Akademie der Wissenschaften, Wolfgang Schuller disait ceci : Die Konzeption dieser Wirtschaftsgeschichte ging von zablreichen Bemerkungen in den Grundrissen von Karl Marx aus und deckte sich, soweit ich das richtig hatte beobachten

3 «Ambassador distressed by U.S. committee charges. Took own life because of "unpleasant publicity" ", Ottawa Evening Journal, 4 avril 1957. Finley ne travailla pas directement avec Norman.

4 "Entretien avec Moses I. Finley ", in M. Finley, Mythe, mémoire, histoire. Les usages du passé, Paris, 1981, p. 258.

5 Voir D. Tompkins, «Max Weber, Karl Polanyi and Moses Finley. Review of Mohammad Nafissi, Ancient Athens and Modern Ideology : Value, Theory and Evidence in Historical Sciences", History and Theory 47 (2008), p. 127.

6 «Le marxisme fait [...] partie intégrante de mon expérience intellectuelle, ce que les Grecs auraient appelé ma paideia» ("Class Struggles », The Listener, 17 août 1967, p. 201).

7 M.I. FinKelstein, compte rendu d'Ettore CiccotTi, La civiltà del mondo antico (Udine, 1935), American Historical Review 42 (1937), p. 37-39. M.I. Finley, Ancient Slavery and Modern Ideology, éd. B. Shaw (Princeton, 1998), p. 154 (et p. 235, n. 50 ; trad. franç. p. 110). Finley invoque aussi Hahn à la p. 232, n. 12 et p. 272. L'essai d'I. HAHN, "Die Anfänge der antiken Gesellschaftsformation in Griechenland und das Problem der sogenannten asiatischen Produktionsweise ", Jahrbuch für Wirtschaftsgeschichte 2 (1971), p. 23-39, fut assez bien reçu dans les pays de l'Est, p. ex. D. Lotze, "Varianten der Produktionsweise in der griechischen Landwirtschaft der archaischen Periode ", in J. Herrmann et I. Sellnow (éd.), Produktivkräfte und Gesellschaftsformation in vorkapitalistischer Zeit, Berlin, 1982, p. 305, n. 8, mais seulement par Finley à l'Ouest.

8 "In realtà, l'impostazione di base del libro del Finley sull'economia antica è esplicitamente antimarxista " (V. Di Benedetto, Filologia e marxismo. Contro le mistificazioni, Naples, [1980], p. 90). 
können, auf weite Strecken mit der Konzeption von Moses Finley und seiner Schule - tant mieux pour Marx, ist man versucht zu sagen?.

Le présent essai se concentre sur les premières étapes de la formation de Finley. Les documents sur les années américaines ne sont pas nombreux, en partie, je pense, parce que Finley savait qu'il était un homme fiché, traité de "stalinien notoire " dans une publication nationale, désigné nommément par le directeur du FBI J. Edgar Hoover en personne ${ }^{10}$, et exclu de pas moins de deux postes d'enseignant. Aux yeux de Roger Baldwin, directeur de l'American Civil Liberties Union, les libertés civiques de Finley n'avaient pas d'importance ${ }^{11}$. Le FBI perquisitionna son domicile et voulut le faire accuser de parjure ${ }^{12}$. Aux États-Unis, bien plus qu'en Angleterre ou en France, être lié au Parti communiste était dangereux, et la prudence s'imposait : on ne laissait pas traîner de documents ${ }^{13}$.

Suivre la trace imprécise de Finley exige le même bricolage qu'il prescrivait aux historiens : construire, à partir de données fragmentaires, un modèle "imparfait, incomplet, grossier, mais qui cependant relie suffisamment les éléments essentiels [...] avec un système de valeurs approprié pour supporter l'analyse comparative, [...] le seul contrôle dont nous disposions ${ }^{14}{ }$. Ex ungue leonem.

\section{La thèse sur Harlan (1927-1929)}

Finkelstein était encore adolescent lorsqu'il écrivit sa thèse sur John Marshall Harlan (1833-1911), un juge de la Cour suprême des États-Unis célèbre pour son refus d'endosser des décisions majoritaires. Harlan était connu pour s'être opposé à la ségrégation raciale, notamment la décision Plessy v. Ferguson en 1896, et pour avoir insisté sur le

9 W. SCHUlLER, "Inhalte Althistorischer Forschung in der DDR ", in I. STARKE (éd.), Elisabeth Charlotte Welskopf und die alte Geschichte in der DDR, Stuttgart, 2005, p. 78-89.

10 J. Edgar Hoover, directeur du FBI, au ministre de la Justice, 12 décembre 1941 (dossier de Franz Boas au FBI, Archives Franz Boas, American Philosophical Society).

11 P. Kuznick, Beyond the Laboratory. Scientists as Political Activists in 1930s America, Chicago, 1987, p. 337, n. 62.

12 Finley, dossier du FBI 100-26441 (reçu grâce au Freedom of Information Act).

13 Pour plus de détails sur le travail de Finley dans les années 1930, voir D. ToMPKINS, "The world of Moses Finkelstein : The year 1939 in M.I. Finley's development as a historian ", in M. Meckler (éd.), Classical Antiquity and the Politics of America, Waco, 2006, p. $95-126$.

14 « On peut construire un modèle, imparfait, incomplet, grossier, mais qui cependant relie suffisamment les éléments essentiels de la structure politique et sociale avec un système de valeurs approprié pour supporter l'analyse comparative. C'est là le seul contrôle dont nous disposions en l'absence de documentation externe " ("The world of Odysseus revisited ", Appendice I de The World of Odysseus, New York 1978, p. 159 [trad. franç. p. 191]). Finley changea de nom en octobre 1946 : j'utiliserai chaque nom selon les circonstances. 
droit de l'individu à une "procédure équitable ${ }^{15}$ ». La thèse n'est pas un ouvrage théorique, mais les causes choisies par Finley montrent qu'il s'intéresse aux préoccupations de Harlan en ce qui concerne la citoyenneté, les droits, la procédure équitable et l'héritage de la loi coutumière (tous points d'importance pour Finley adulte), et qu'il émet des réserves sur l'argumentation de Harlan ${ }^{16}$. Par une ironie du sort, il invoquerait ses propres droits devant une commission d'enquête parlementaire un quart de siècle plus tard. Par une même ironie, l'opinion majoritaire du petit-fils de Harlan dans la cause Yates $v$. United States (1957) atténua le risque d'être poursuivi pour activisme politique $^{17}$. Mais Finley avait quitté le pays. L' « encyclopédiste " : le début des activités à gauche (1930-1934).

\section{"Les paysans se réjouissent de l'ordre de Staline »}

En 1927, le père de Finkelstein l'aida à trouver une situation au service juridique de General Motors. Il s'ennuya, partit et ne tarda pas à entamer des études de premier cycle à l'université Columbia, et il travailla comme rédacteur et bibliographe à l'Encyclopaedia of the Social Sciences $^{18}$. En août 1932, il écrivit à Fritz Heichelheim après avoir vérifié l'article de ce dernier sur le régime foncier dans l'Antiquité ; ce fut le début d'une enquête sur les "problèmes de la terre " qui l'occuperait toute sa vie. Les archives de l'Encyclopaedia, données par l'éditeur à la bibliothèque du Hampshire College (Massachusetts), montrent que le même mois où Finkelstein écrivait à Heichelheim, Peter Struve à Paris soumettait un long essai sur le "régime foncier en Russie ", condamnant la politique agricole de Staline. Peter Struve voyait dans la " dékoulakisation " une forme de "cruauté médiévale [...]. Une famine effroyable, comme la Russie n'en avait jamais connue ${ }^{19}$ ». Mais ce réquisitoire ne parut jamais dans l'Encyclopaedia:

15 M. Finkelstein, Justice Harlan on Personal Rights with Special Attention to Due Process of Law, thèse, Master of Arts en sciences politiques, université Columbia, 1929.

16 Pour plus de détails sur la thèse de Finley, voir D. Tompkins, " Moses Finkelstein and the american scene : The political formation of Moses Finley, 1932-1955 ", in W.V. HarRIs (éd.), Moses Finley and Politics, Leyde et Boston, 2013, p. 5-30.

17 Voir A. Sutherland Jr., "The Supreme Court, 1956 Term », Harvard Law Review 714 (1957), p. 88-89.

18 «Mon père était chauffeur et je conduisais bien. Il avait de grandes aspirations banales. Je serais avocat et le président d'une société. [...] Mon père me trouva un travail au service juridique de General Motors, mais je m'en allai au bout de six mois. Je me rebellai et cela provoqua une certaine tension qui ne se termina jamais jusqu'à sa mort à l'âge de 90 ans. Il avait un côté très patriarcal. " "Cela a-t-il un rapport avec ce qui vous intéresse dans The World of Odysseus? " "Je ne sais pas. Je n'ai jamais pu faire preuve de beaucoup d'introspection sur ce genre de chose " (R. WInKLER, "A conversation with Moses Finley ", 1980, Archives Finley).

19 Manuscrit dactylographié, archives de l'Encyclopaedia of the Social Sciences, Hampshire College. 
il ne figure que dans les archives. Les éditeurs le remplacèrent par un éloge de Staline et de son prétendu " ordre contre la collectivisation forcée ", qui s'inspirait d'un récit fallacieux de Walter Duranty dans le New York Times, "Peasants rejoice over Stalin $\operatorname{order}^{20} »$. Aucune raison n'est avancée. Si les éditeurs jugeaient Duranty plus crédible, ils se trompaient : il s'agissait d'une des nombreuses tromperies dans une carrière qui valurent à leur auteur le prix Pulitzer en 1936 et, plus tard, la disgrâce ${ }^{21}$.

Finkelstein savait-il que ses collègues avaient fait de la famine de masse une festa contadina? Nous ne pouvons en être sûrs. Mais à l'âge de vingt ans, il était sur le point de s'engager dans une réécriture majeure et politisée de l'histoire.

\section{Bernhard Stern, «stalinien notoire »}

La censure de Struve par les éditeurs de l'Encyclopaedia trahit peut-être un a priori politique. "Marx était devenu à la mode ", dit Alvin Johnson, éditeur associé de l'Encyclopaedia, rappelant qu'il avait engagé de brillants auteurs, dont Max Lerner, Lewis Corey, Louis Hacker, Herbert Solow et Bernhard Stern, tous actifs à gauche à des degrés divers ${ }^{22}$. L'un d'entre eux fut-il tenté d'éliminer l'essai de Struve ? Nous l'ignorons. Cependant le nom de Bernhard Stern se détache. Sept ans plus tard, après que Finkelstein eut été promu d'éditeur et réviseur à " véritable organisateur " et «stalinien notoire ", son nom se trouva associé à celui de Stern dans des accusations d'agitation de gauche à Columbia ${ }^{23}$.

Stern fut un pionnier de l'anthropologie sociale, influençant Robert Merton ${ }^{24}$. Il fonda la revue marxiste Science and Society, et en 1932 déjà il connaissait Lionel Trilling, Meyer Schapiro, Granville Hicks, Max Lerner et d'autres. Il fut aussi un " recruteur " très actif, bien que souvent anonyme, pour le Parti communiste, ainsi que l'une des deux relations de Finkelstein qui louèrent Le Matérialisme dialectique et

20 New York Times, 8 mars 1930.

21 Voir S. TAYLOR, Stalin's Apologist. Walter Duranty: The New York Times's Man in Moscow, New York, 1990.

22 Dans son entretien de 1980 avec Winkler (voir supra n. 18), Finley dit que Louis Hacker fournit les contacts qui lui valurent des propositions de poste à Christ Church, Oxford, et à Jesus College, Cambridge, en 1953.

23 B. Stolberg, " "Innocent front" : Catspaws of Communism ", Washington Post, 2 décembre 1939, p. 11 ; The New Leader, 27 avril 1940, parlait de Finkelstein comme d'un "stalinien bien connu ", attaquant aussi Mary Dublin, la future femme de Leon Keyserling (conseiller économique de Truman), et I.F. Stone, mais pas Stern. Un ancien étudiant de Finley, Daniel Bell, devint éditeur de cette publication en 1941.

24 On trouve dans les archives Robert Merton à l'université Columbia nombre de matériaux sur Stern, dont des documents montrant que les professeurs réussirent à protéger le poste de Stern sous l'ère McCarthy. Sur les études médicales de Stern, voir Samuel W. BLOOM, The Word as Scalpel. A History of Medical Sociology, New York, 2002. 
le matérialisme historique de Staline (1938) dans des revues universitaires américaines ${ }^{25}$. Son stalinisme exaspérait les chercheurs de l'école de Francfort ${ }^{26}$. Il se peut qu'il ait recruté Finkelstein, comme il avait recruté Hicks ${ }^{27}$.

\section{Les condisciples de Finkelstein}

Au département d'études classiques de Columbia, les plus proches amis de Finkelstein étaient Meyer Reinhold et Naphtali Lewis ${ }^{28}$. En dehors du département, ses condisciples en histoire et en sciences sociales s'appelaient Ben Nelson, Charles Trinkhaus, Samuel Coleman et Daniel Thorner. Finley se souvient : "À l'université Columbia, j’ai commencé par apprendre l'histoire ancienne [...]. Ce furent des années de tension considérable [...], les cours et les séminaires étaient [...] enfermés dans une tour d'ivoire. [...] Je veux parler [...] du déphasage [de nos professeurs] dans leur travail en tant qu'historiens. On aurait pu donner les mêmes cours et les mêmes séminaires - et ce fut sans aucun doute le cas - une génération auparavant, avant la Première Guerre mondiale. [...] Alors nous avons décidé de nous-mêmes de chercher dans les livres ce que, à notre avis, les cours et les séminaires ne nous donnaient pas. Nous lisions et discutions Marc Bloch et Henri Pirenne, Max Weber, Veblen et les freudiens, [...] Marx et les marxistes [...] pas seulement Le Capital, pas même surtout Le Capital, mais aussi les ouvrages marxistes historiques et théoriques. Le marxisme fait donc partie intégrante de mon expérience intellectuelle, [...] de ma paideia. Marx [...] m'ôta de la tête que l'étude de l'histoire est une activité autonome $[\ldots]^{29}$."

La plupart de ces jeunes gens était pauvres. Les parents de Daniel Thorner invitaient parfois des étudiants à dîner ; les Finkelstein invitaient des amis chez eux le dimanche soir, pour écouter des disques de Gilbert et Sullivan ou peut-être parler politique, formant ce "groupe d'étude marxiste " qui prit plus tard de si inquiétantes

25 B. STERN, compte rendu de Leninism : Selected Writings by Joseph Stalin, American Economic Review 33 (1943), p. 395-397. L'autre relation était vraisemblablement V. KAZAKEVITCH (voir infra note 72), "Social sciences in the Soviet Union ", American Sociological Review 9 (1944), p. 312-318.

26 Leo Lowenthal, lettre à Horkheimer, in LowenTHAL, Critical Theory and Frankfurt Theorists : Lectures, Correspondence, Conversations, New Brunswick (N.J.), 1988, p. 212.

27 Lorsqu'il raconte qu'il a décidé, lors d'un dîner détendu au début des années trente, d'adhérer au parti, Hicks ne dit pas un mot de Stern : Part of the Truth. An Autobiography, New York, 1965, p. 128. Stern reconnut son rôle dans un témoignage devant un comité professoral de Columbia (rapport professoral au président Grayson Kirk, 3 juin 1953 [Archives Merton]).

28 W. Briggs, "Meyer Reinhold ", Gnomon 75 (2003), p. 380-382, et communications personnelles, 2006.

29 "Class Struggles », p. 201-202. 
proportions dans le témoignage de Karl August Wittfogel ${ }^{30}$. Finley travaillait à temps partiel, enseignant l'histoire au City College, où la présence du Parti communiste chez les professeurs d'histoire se faisait particulièrement sentir. Un étudiant de Finley, Daniel Bell, plus tard sociologue de premier plan, fut si influencé qu'il changea son domaine de recherche de premier cycle pour l'histoire ancienne, encore que les rapports se soient détériorés lorsque les deux hommes devinrent adversaires au début des années quarante. Il y eut plus tard un rapprochement émouvant ${ }^{31}$.

\section{Reinhold et Rostovtzeff (1946)}

En 1965, le grand ami de Finkelstein Meyer Reinhold démissionna de son poste au Brooklyn College, afin, dit-on, de ne pas répondre à des questions sur ses amis ${ }^{32}$. Apparemment dégagé de toute participation à des organisations de gauche, Reinhold « dévia » seulement en publiant sa remarquable " Critique of Rostovtzeff » dans Science and Society ${ }^{33}$ : "En confrontant les opinions de Rostovtzeff sur les forces politiques, économiques, culturelles et psychologiques dans la causalité historique avec les idées des sciences économiques contemporaines et, marginalement, la théorie marxiste, Reinhold trouva que Rostovtzeff était magistral dans sa présentation des preuves documentaires mais qu'il laissait à désirer du point de vue méthodologique et conceptuel ${ }^{34}$. » Reinhold

30 United States Congress, Senate, Committee on the Judiciary, Institute of Pacific Relations : Hearings before the Subcommittee to Investigate the Administration of the Internal Security Act and Other Internal Security laws of the Committee on the Judiciary, Washington, 1951-1952, p. 318.

31 Voir la lettre de Bell à Elisabeth Sifton (Archives Finley, 28 octobre 1980), où il la remercie de lui avoir envoyé avant publication un exemplaire d'Ancient Slavery and Modern Ideology de Finley. Bell dit que Finley fut son professeur et son ami mais, plus tard, " un adversaire politique dans les vieilles querelles des milieux intellectuels new-yorkais ». Les Archives renferment aussi des lettres à Allan Bloom où Bell critique ce dernier à cause de son compte rendu négatif d'Ancient Slavery and Modern Ideology dans le New York Times (11 janvier 1981), et la réponse de Bloom qui reproche à Bell son « mccarthysme très personnel » et sa "petite vanité servile devant les sièges du pouvoir » 22 janvier-10 février 1981). Dans une lettre à Finley (20 février 1981), Bell disait ceci : "Bloom est un de ces straussiens qui se sont pris pour les tuteurs occultes des puissants (ce qui apparaît assez nettement dans [...] les échanges extraordinaires de Strauss avec Kojeve), ou pour les jésuites à la cour d'Espagne [...]. Quant à la vieille bagarre entre Boas et Dewey, autre temps, autres mœurs. "

32 BRIGGS, " Meyer Reinhold », p. 381.

33 "Historian of the classical world : A critique of Rostovtzeff ", Science and Society 10 (1946), p. 361-391 = M. Reinhold, Studies in Classical History and Society, New York, 2002, p. $82-100$.

34 W. HaAse et I. Horowitz, "In memoriam Meyer Reinhold (1909-2002)", International Journal of the Classical Tradition 9 (2002), p. 3 ; M. FinLeY, compte rendu d'Arnaldo 
n'écrivit rien de semblable auparavant ou plus tard. Le nom de Finkelstein n'apparaît nulle part - omission prudente ? - mais sa présence se fait sentir lorsque son ami s'appuie sur des ouvrages qui avaient la faveur de Finkelstein (l'Agrarverhältnisse de Weber et Gunnar Mickwitz sur le "rationalisme économique ${ }^{35}$ »), et lorsqu'il décide d'écrire pour la revue de gauche du collègue de Finkelstein, Bernhard Stern. Étant donné la tendance de l'époque, ce choix aurait profondément déplu aux supérieurs de Reinhold au Brooklyn College ${ }^{36}$.

\section{L'École de Francfort (1934-1947)}

Si le curriculum vitae officiel de Finley dit avec justesse que ce dernier fut employé de 1937 à 1939 par l'Institute of Social Research de l'École de Francfort à New York, Finley travailla aussi comme traducteur et rédacteur à temps partiel pendant plus de dix ans, et son aide fut précieuse pour la communication avec le public américain ${ }^{37}$. Pour Finkelstein, l'expérience fut formatrice : "Ces gens furent les premiers, je crois, à être vraiment engagés dans la sociologie de la culture, avec une profondeur et une portée très considérables [...]. Ils jouèrent un rôle très important dans mon éducation ${ }^{38}$. "

Lorsque Finley retourna dans le monde universitaire en 1947, à l'âge de 35 ans, ses références étaient Westermann, Horkheimer et Paul Lazarsfeld ${ }^{39}$. Herbert Marcuse,

Momigliano, Terzo contributo alla storia degli studi classici e del mondo antico, History and Theory, 7 (1968), p. 357.

35 G. MiскWITZ, "Economic rationalism in graeco-roman agriculture ", English Historical Review 52 (1937), p. 577-589. Cité par ReInHold, Science and Society, p. $368=$ Studies, p. 85 ; et par M. Finley, The Ancient Economy, Berkeley, 1973, p. 233, n. 43 (trad. franç. p. 146). Agrarverhältnisse : voir ReInHold, Science and Society, p. 387 = Studies, p. 98. Finley mentionna l'Agrarverhältnisse de Weber pour la première fois à l'âge de 22 ans : "Emporos, Naukleros and Kapelos ", Classical Philology 30 (1935), p. 320.

36 BRIGGS, "Meyer Reinhold ", p. 381. Dans son témoignage devant l'Internal Security Subcommittee (supra, note 30) le 11 mars 1953, Harry Gideonse, président du Brooklyn College, attaqua violemment les communistes de l'université, s'en prenant nommément à Moses Finkelstein pour de prétendues négligences quatorze ans auparavant. http://academic.brooklyn.cuny.edu./english/melani/bc/senate1952/gideonse.html.

37 "The early development of M.I. Finley's Thought : The Heichelheim dossier ", Athenaeum 81 (1993), p. 196.

38 Winkler (supra, note 18).

39 Horkheimer, 28 mai 1947, note que Finley, outre son emploi officiel, de 1937 à 1939, " fit aussi régulièrement des comptes rendus d'ouvrages sur l'histoire ancienne [...], avant, pendant et après cette époque. [...] Finley [...] travaillait [...] en étroite collaboration en sa double qualité de traducteur et de rédacteur. Il lui incombait de préparer la traduction en anglais de livres, articles et conférences en allemand sur la philosophie, la théorie politique, la sociologie, l'histoire, le droit et la littérature. En tant que rédacteur, il lui fallait non seulement fournir des traductions exactes mais aussi [...] participer à la révision des textes afin de les rendre aussi clairs et intelligibles que possible pour le lecteur américain. 
un autre membre de l'institut, exerça aussi une influence, et Finkelstein fit l'éloge du Raison et révolution de Marcuse à sa parution en $1941^{40}$. En 1971 Finley dit à Quentin Skinner que l'essai de Horkheimer Egoismus und die Freiheitsbewegung (1936) soustendait sa conférence inaugurale, The Ancestral Constitution. Finley se faisait l'écho de Horkheimer en critiquant " les historiens [qui] se préoccupent peu de la psychologie des peuples qu'ils étudient, de ce qu'on appelle en France les structures mentales [...]. Je ne parviens pas à comprendre pourquoi les facteurs psychologiques ont droit de cité [dans l'histoire de la religion] alors qu'ils sont exclus lorsqu'il s'agit d'étudier les comportements politiques ${ }^{41} »$. En fait, The Ancestral Constitution fourmille d'allusions au passé politique de Finley ${ }^{42}$.

Pour qui étudie l'usage que Finley fait de Marx, c'est important. Horkheimer, Theodor Adorno et d'autres membres de l'institut revendiquaient des positions marxis-

Quiconque connaît bien la terminologie philosophique allemande se rendra compte des difficultés d'une telle mission. " Selon Horkheimer, Finley joua un rôle majeur étant donné que l'Institut " effectuait le passage du contexte allemand au contexte américain [parce que Finley était compétent en histoire, en sociologie et en philosophie et qu'il avait] le chic de la présentation limpide [...]. » (Archives Lewis Webster Jones, université Rutgers).

40 M. Finkelstein, compte rendu de B. Farrington, Science and Politics in the Ancient World (Oxford, 1941), Zeitschrift zur Sozialforschung 9 (1940), p. 502.

41 Finley, The Use and Abuse of History, New York, 1975, p. 46 (trad. franç. p. 228), et la lettre de Finley à Quentin Skinner, 13 juillet 1971 : "Si vous vous sentez d'attaque pour un bonne tranche d'allemand plutôt hégélien, vous pourriez trouver quelque intérêt à Max HoRKHEIMER, "Egoismus und die Freiheitsbewegung ", Zeitschrift für Sozialforschung 5 (1936). Je l'ai lu lors de sa parution (je travaillais alors pour l'Institut à New York), et j'ai le sentiment, en dernière analyse, que c'est ce qui m'a poussé à m'intéresser à cette idée. Je ne l'ai pas cité dans ma conférence inaugurale parce qu'en le relisant pour l'occasion, je m'aperçus qu'il partait rapidement vers de tout autres sujets " (Archives Finley).

42 Parmi les autres thèmes des années trente dans cette conférence : le désir de tous les partis politiques américains de prendre Thomas Jefferson comme modèle, suggérant, mais sans le mentionner explicitement, le rôle de Finley dans la mise à profit de Jefferson, Use and Abuse, p. 44-46 (trad. franç. p. 222-224) ; éloge de Staughton Lynd, leader de la nouvelle gauche américaine, un rappel que le père de Lynd, Robert, avait été un camarade de Finley dans les luttes politiques des années trente, Use and Abuse, p. 45 (trad. franç. p. 226) ; et un renvoi à Marc Bloch lorsqu'il se fait l'avocat d'une histoire professionnelle, Use and Abuse, p. 58 (trad. franç. p. 249) : «Dans le vocabulaire courant, dit Marc Bloch, les origines sont un commencement qui explique. Pis encore : qui suffit à expliquer. [...] La simplicité est $[\ldots]$ la clé $[. .$.$] du succès populaire [...]. » Une décennie auparavant,$ Finley invoquait Bloch et sa remarque sur les " féodalités exotiques " pour critiquer les historiens qui faisaient d'un concept historique important une métaphore grossière ("The Mycenaean tablets and economic history ", Economic History Review 10 [1957], p. 128-141) ; M. Bloch, La société féodale, Paris, 2002, p. 603. Comme nous le rappelle ce passage, dans les années trente Finley avait « lu et discuté Marc Bloch et Henri Pirenne, Max Weber, Veblen et les freudiens [...]» («Class Struggles », p. 201-202). 
tes mais non staliniennes ${ }^{43}$. Au même moment, deux des autres relations de Finkelstein, Stern et Kazakevitch, défendaient dans des publications américaines le fameux texte de Staline, Le Matérialisme dialectique et le matérialisme historique ${ }^{44}$.

\section{L'American Committee for Democracy and Intellectual Freedom (1938-1942)}

Parallèlement à ses études universitaires, au travail pour l'Institute of Social Research et à l'enseignement au City College, Finkelstein s'embarqua dans son aventure la plus lourde de conséquences, une association politique avec Franz Boas. En 1980 il en rappela certains détails - mais en omit d'autres : "Je ne me suis jamais engagé dans la politique classique. J'ai toujours été en marge. En 1938, Nature publia, par provocation délibérée, un article d'un physicien nazi agressif sur "la physique allemande et la physique juive", une critique véhémente d'Einstein ${ }^{45}$. Trois d'entre nous, assis dans un restaurant self-service, décidèrent qu'il fallait faire quelque chose. L'un de nous était anthropologue. Il se rendit chez Franz Boas, qui donna son accord, et il rédigea une courte déclaration. Puis, nous avons fait un coup assez fou : nous avons mis la main sur des listes de professeurs d'environ 1200 universités, nous avons envoyé la déclaration avec la signature de Boas et nous avons rassemblé 1500 noms. Nous n'étions qu'une petite bande d'étudiants, ne connaissant rien à rien, mais nous avons publié un communiqué de presse avec tous les noms et nous avons fait la première page du New York Times ${ }^{46}$. Boas dit alors : "On ne va pas en rester là, non ?" Il s'occupa de trouver de l'argent et mit sur pied l'American Committee for Democracy and Intellectual Freedom [...]. Un des plus jeunes membres

43 Selon Marcuse, la propriété d'État risquait de devenir tout bonnement une " nouvelle forme d'assujettissement des individus à une universalité hypostasiée "; Horkheimer dit que " cette image à l'horizon dont parlent toujours les politiciens socialistes m'a tout l'air d'être un mirage ». Voir K. Anderson, "On Hegel and the rise of social theory: A critical appreciation of Herbert Marcuse's Reason and Revolution, fifty years later ", Sociological Theory 11 (1993), p. 249-250, renvoyant à Reason and Revolution, p. 283. M. Horkheimer, "The authoritarian state ", in A. Arato \& E. Gebhardt (éd.), The Essential Frankfurt School Reader, New York, 1978, p. 115.

44 Voir supra, note 25.

45 J. STARK, "The pragmatic and the dogmatic spirit in physics ", Nature, 30 avril 1938, p. $770-772$.

46 Un éditorial non signé, "Nordic science ", parut dans le New York Times du 29 mai 1938. Le comité Boas y répondit par " Nazi’s conception of science scored " 11 décembre 1938). Parmi les autres succès de Finkelstein avec la presse on relèvera : "Schools rebuked on racial errors" (New York Times, 17 août 1939) ; "Aliens defended in "race" dispute", qui répondait à la brochure antisémite de l'eugéniste Harry Laughlin sur l'immigration et distribuée par la Chambre de commerce de New York (23 juillet 1939) ; et encore sur cette chambre "Report on Schools scored as "Fascist" " (3 octobre 1939). 
s'appelait J. Robert Oppenheimer. Nous fîmes du bruit, comme les groupes de pression, et puis nous nous occupâmes de la commission Dies ${ }^{47}$. C'est alors que l'on commença à parler de "façade communiste" et, vu la structure du comité, la façade communiste ne pouvait être que moi. "Oh là là ! Tous les autres se faisaient simplement mener par le bout du nez. Eh bien, quand les États-Unis entrèrent en guerre, le comité n'avait plus de raison d'être et il fut liquidé. Mais à ce moment j'étais déjà bien étiqueté ${ }^{48}$. "

Par circonspection peut-être, Finley ne cite pas le nom de l'" anthropologue " qui le présenta à Boas. Le nom qui vient surtout à l'esprit est celui de Bernhard Stern, un ami de Boas. Mais Stern n'était pas seulement " anthropologue ». Bien que Finley ne cite jamais son nom dans ses écrits, Stern avait travaillé avec Finkelstein sur l'Encyclopaedia en 1932, et il lui fut directement associé en 1941 comme leader stalinien à Columbia $^{49}$. Si l'intermédiaire entre Finley et Boas n'était pas un universitaire quelconque mais le fondateur de Science and Society, un collaborateur à New Masses, et un recruteur majeur du Parti, notre compréhension des origines du comité et de la pensée de Moses Finley en 1980 se complique. Le flou artistique de Finley en 1980 ne laisse pas d'intriguer ${ }^{50}$.

\section{Boas et Finkelstein contre Dewey et Hook}

De 1938 jusqu’à la mort de Boas en 1942, Finkelstein fut le principal agent et publiciste du comité : il plaça des articles dans le New York Times, organisa des événements et établit des réseaux dans tout le pays. Le comité, en grande partie un produit du militantisme de la guerre d'Espagne, était l'exemple d'une politique de front populaire, adoptant des positions bien arrêtées sur l'immigration juive, les libertés civiques, les manuels scolaires, la liberté universitaire et le racisme en Amérique. Finkelstein écrivit

$47 \mathrm{La}$ " commission Dies » était la Commission des affaires antiaméricaines de la Chambre des représentants, dont le président était Martin Dies (républicain, Texas), 1936-1944.

48 Winkler (supra, note 18).

49 Stolberg (supra, note 24). Stolberg fut un allié de Stanley Hook dans les luttes anticommunistes à New York et à Columbia.

50 Il faudrait que nous ayons une idée claire et précise de la nature du contrôle exercé par le Parti sur les groupes de type front populaire. À l'Ouest, les citoyens "convertis " pouvaient perdre la foi, cela arriva, et s'en aller, ce qui compliquait les tentatives de Moscou pour contrôler les groupes " de façade ». Des documents de Moscou révèlent les déceptions causées parfois par des Américains imprévisibles : le NKID à la vOKS, 28 juin 1937 : "Nous attirons votre attention sur le paragraphe relatif à la nécessité de pousser l'American Russian Institute à se débarrasser de Dewey qui a dévié de la ligne du Parti. » Les "nombreuses mentions » de l'Américain Corliss Lamont " dans les dossiers soviétiques sont un mélange de méfiance et de flatterie " (Svetlana Chervonnaya, communication personnelle, 2012). 
un pamphlet brillant contre le racisme ${ }^{51}$. Le comité comptait parmi ses membres des professeurs de Columbia aussi distingués que Robert Lynd, Ruth Benedict, Wesley Mitchell, Howard Urey et Leslie Dunn, des savants qui, à l'origine, avaient uni leurs forces pendant la guerre d'Espagne ${ }^{52}$.

Les dossiers du comité révèlent une prise de conscience du traitement réservé à Trotsky, des procès-spectacles de Moscou, et des injustices de la guerre d'Espagne. Boas s'exprima ouvertement sur le manque de droits sous Staline, mais il donnait la priorité à la lutte contre le fascisme.

Alors qu'ils venaient tout juste de défendre Trotsky à Mexico, le philosophe Sidney Hook et John Dewey, un vieil ami de Boas, mirent en avant la lutte contre tous les totalitarismes (à l'exception de Trotsky, comme Emma Goldman le fit remarquer à Dewey). Leur Committee for Cultural Freedom (CCF) deviendrait le terreau du futur néo-conservatisme américain ${ }^{53}$. Boas avait quatre-vingts ans et Dewey soixante-dixneuf. La méthode de Hook en politique fut toujours personnelle et souvent déplaisante, par exemple la dénonciation de collègues de gauche, et il contribua à l'hostilité croissante et à la méfiance entre les deux hommes ${ }^{54}$.

Dewey et Hook se concentrèrent sur les maux de Staline, sans prêter attention au travail du comité de Boas sur le racisme, l'antisémitisme, l'immigration et l'éducation. Dans la panique qui suivit le pacte germano-soviétique en août 1939, il n'en fallait pas plus. Le slogan "Finkelstein le stalinien », une invention de Benjamin Stolberg, un associé de Dewey, se répandit rapidement. Roger Baldwin, principal défenseur des

51 Il s'agit de Can You Name Them?, American Committee for Democracy and Intellectual Freedom, 1939.

52 American Philosophical Society, Archives L. C. Dunn.

53 Le 10 janvier 1938, Emma Goldman écrivait à Dewey : "Je ne peux partager les sentiments qui vous ont motivés, vous et votre comité, à défendre Léon Trotzky [sic], car je sais que lorsqu'il était au pouvoir il a fait exactement la même chose que son grand ennemi actuel, Staline, et qu'il ferait preuve de la même inhumanité envers ses adversaires s'il était à nouveau au pouvoir. " (Archives Emma Goldman, Tamiment Library, université de New York ; également dans l'édition électronique de The Correspondence of John Dewey, 1871-1952. Carbondale et Edwardsville, volume 2 : 1919-1939).

54 Hook comme délateur : voir le rapport de Robert Morris, enquêteur pour le corps législatif de l'État de New York, dans les dossiers (Albany) de l'Investigation into the Educational System of the State of New York Pursuant to Joint Resolution of the Senate and Assembly Adopted March 29, 1940 : «Hook fut très bien disposé et très élogieux quant au travail de la commission. Il donna de nombreux exemples de ce que les communistes avaient fait à différentes réunions où il était présent [...]. Il avait l'impression que Robert Gessner, du département d'anglais de l'université de New York, était sans aucun doute membre du parti. À propos de Gessner, il déclara qu'il avait écrit un article pour New Masses, il y a environ six ans, où il affirmait que si quelque chose convenait à Earl Browder [secrétaire général du Parti communiste américain], cela lui convenait aussi [...]. Quant aux gens de Hunter College, Hook indiqua que Rees, peut-être, était un ex-membre du parti. [...] Il [Hook] promit de rechercher des informations susceptibles de nous intéresser. " 
libertés civiques aux États-Unis, se lava les mains des communistes, et de Finkelstein en particulier ${ }^{55}$; pendant une vingtaine d'années, les instances du monde enseignant américain s'en tiendraient à ce dogme : les communistes n'étaient pas aptes à enseigner ${ }^{56}$. En 1939 l'American Jewish Committee retira son soutien à Boas au profit de Dewey ${ }^{57}$; en mai de la même année, l'État de New York adopta une loi demandant le renvoi des enseignants communistes ${ }^{58}$, et le 28 juin 1940 le gouvernement fédéral adopta le Smith Act, qui sanctionnait pénalement les "buts " et la " défense " des communistes ${ }^{59}$.

\section{Films for Democracy}

Dans une décennie où Hollywood refusa notoirement de critiquer les dictateurs, Blockade de Walter Wanger (1938) fut le « seul film à s'interroger vraiment sur les objectifs [de la guerre d'Espagne] ${ }^{60}$ ". Et Wanger, bien connu pour son "indépendance ", devint un personnage clé de Films for Democracy, un groupe soutenu par Finkelstein et Boas, et qui visait à " produire des films pour 100000 dollars ou moins, en utilisant les techniques d'Hollywood pour séduire le plus large public possible, et persuader les studios de les distribuer ». Films for Democracy sombra après avoir été accusé d'être une "façade communiste " en 1939. Il contribua à promouvoir non seulement Blockade mais aussi Juarez de Warner Brothers. Gardner Jackson, un homme du New Deal à Washington, généreux, bavard, crédule et porté sur la boisson, se remémora Finkelstein des décennies plus tard : "Une des choses qui m'attirèrent [...] fut un comité créé sans doute pour peser sur Hollywood afin que soient produits des films qui serviraient

55 Roger Baldwin, fondateur et directeur de l'American Civil Liberties Union, en avril 1942 : "Je sais beaucoup de choses sur l'American Committee for Democracy and Intellectual Freedom [...]. Il ne fait aucun doute que le comité [...] a une orientation pro-communiste marquée et que son secrétaire appointé, Finkelstein, est soit un membre du Parti communiste soit un proche sympathisant. " (P. KuZnicK, Beyond the Laboratory. Scientists as Political Activists in 1930s America, Chicago, 1987, p. 337, n. 62).

56 Voir l'important discours de 1939 : S. Hook, "Academic freedom and the "Trojan horse" ", Bulletin of the American Association of University Professors 25, 5 (décembre 1939), p. 550-555.

57 KuZniCK, Beyond the Laboratory, p. 217.

58 C'était la loi Devany, réclamant le renvoi des enseignants qui " enseignent ou professent des croyances dans toute doctrine prônant le renversement du gouvernement par la force ». (New York Times, 29 mai 1939 : « [Governor Herbert] Lehman signs Bill to bar subversion in public service ").

59 Voir Sutherland (supra, note 18).

60 M. Bernstein, Walter Wanger: Independent Producer, Berkeley et Los Angeles, 1994, p. 134. Sur les années trente: T. DOHERTY, Hollywood and Hitler : 1933-1939, BerkeleyLos Angeles, 2013 ; B. Unwand, The Collaboration. Hollywood's Pact with Hitler, Cambridge (Mass.), 2013. 
les intérêts de la démocratie. Si seulement je pouvais me rappeler le nom du type qui m’a persuadé ! C'était un gars des plus charmeurs, un jeune professeur de l'université Columbia. Films for Democracy, c'est ainsi que nous nous appelions [...]. Le groupe contribua au financement d'un film. [...] Un type de chez Warner Bros vint me voir en secret et me dit qu'il travaillait avec ce jeune professeur charmeur - si seulement je pouvais me rappeler son nom ! Ils allaient faire un film [...] sur Juarez. [...] C'était un sacré film, avec Paul Muni ; le film aurait une autre importance plus tard [... ${ }^{61}$."

Presque totalement incohérents, les scénarios de Juarez et de Blockade montrent que leurs auteurs sentaient le regard des censeurs par-dessus leurs épaules ${ }^{62}$. Nous savons peu de chose sur le travail de Finkelstein avec Films for Democracy, mais les souvenirs de Jackson nous rappellent la variété de ses activités à la fin des années trente.

\section{Le Russian War Relief (RWR) ${ }^{63}$}

En 1942, Finkelstein ne fut pas en mesure de servir pendant la guerre car il fut réformé par l'armée américaine à cause d'une " psychonévrose grave », diagnostic peu crédible qui trahit peut-être une aversion pour les gens de gauche et les Juifs ${ }^{64}$. Après les auditions de la commission Rapp-Coudert du corps législatif de New York (1941-1942), qui coûtèrent à Finkelstein et à trente de ses collègues leur place au City College ${ }^{65}$, il

61 Reminiscences of Gardner Jackson. Oral History (1963), Columbia Center for Oral History. Voir le beau chapitre de M. KEMPTON ("The dry bones ») sur Jackson et Lee Pressman dans Part of Our Time. Some Monuments and Ruins of the Thirties, New York, 1957, p. 37-82. Un document anonyme, Films for Democracy, conservé dans les Archives Jackson (bibliothèque Franklin D. Roosevelt, Hyde Park, N.Y.) expose la mission du groupe : "Encourager les films qui soutiennent la démocratie américaine, les libertés civiques et la paix ; qui favorisent une meilleure compréhension et de meilleures relations entre les groupes raciaux et religieux ; qui donnent une image exacte, non déformée aussi bien que socialement utile de la situation contemporaine. S'opposer aux tendances fascistes, aux attaques contre le syndicalisme, et aux films contraires à la Déclaration des droits. "

62 Le censeur : Joseph Breen, du Motion Picture Production Code, célèbre pour avoir dit à propos d'Hollywood que " quatre-ving-dix-neuf pour cent de ces gens sont des Juifs [...]. Ils sont, sans doute, l'écume du monde. " (S. Eyman, Lion of Hollywood: The Life and Legend of Louis B. Mayer, New York, 2005, p. 343).

63 Sur le Russian War Relief, voir Fr. NaIden, "Finley's War Years ", American Journal of Philology, à paraitre. Un ami intime de Finley, C. Whittaker, a dit quelle était l'opinion admise au Royaume-Uni : "Les années de guerre sont comme une lacune dans la carrière de Finley [...]. Son attention fut détournée vers ce qu'il appelait les "agences de secours de guerre”. » (C. WhitTaker, « Moses Finley 1912-1986 », Proceedings of the British Academy 94 [1997], p. 462).

64 Dossier de Finley au FBI.

65 E. Schrecker, No Ivory Tower. McCarthyism and the Universities, New York, 1986, p. 81. 
enseigna à la Worker's School parrainée par le Parti, mais il travailla surtout pour le Russian War Relief, qu'il contribua à mettre sur pied et qui envoya pour cinquante millions de dollars de marchandises (c.-à-d. plus de cinq cent millions de dollars d'aujourd'hui), aux shtetls d'Europe orientale. À la fin de la guerre il était devenu directeur de campagne national ${ }^{66}$.

Le RWR suscita l'enthousiasme de la population juive américaine, et reçut des dons substantiels : dans un excellent essai, Fred Naiden rapporte que les donateurs pauvres "se servirent de toutes sortes de charrettes et de tout ce qui avait des roues". L'ambassadeur Maxim Litvinov devint un collecteur de fonds efficace et il aida aussi Finkelstein dans ses rapports avec les bureaucrates soviétiques et les comités juifs. Finkelstein permit aussi de recruter des orateurs comme Albert Einstein, qu'il conduisit à la tribune du Madison Square Garden et dont il fut la plume ${ }^{67}$. Il accompagna aussi le célèbre acteur du théâtre yiddish Solomon Mikhoels lors de sa rencontre avec Charlie Chaplin ${ }^{68}$. Naiden dit en conclusion que Finkelstein « en savait peut-être plus que n'importe qui sur la situation malheureuse du judaïsme mondial - plus que le Département d'État, qui ne tenait pas compte des informations données par les partisans, peut-être plus que le gouvernement soviétique, qui connaissait la situation de son propre peuple, mais savait peu de chose sur les Juifs américains inquiets qui avaient des parents en URSS. Finkelstein connaissait la situation dans les deux pays. Avec son équipe il était de ceux à qui l'on demandait si un parent se trouvait parmi les deux millions de Juifs dont le Jewish Council savait qu'ils avaient été évacués devant la progression des nazis ${ }^{69}$. Trois cent quarante-sept Juifs qui ne furent pas évacués de Riga survécurent, et Finkelstein et Levine, son patron, furent les premiers Américains à connaître leurs noms ${ }^{70}$.

\section{L'American Russian Institute (ARI)}

À l'été 1946, des organisations favorables aux soviétiques se lancèrent dans une campagne massive de publicité et de recrutement ${ }^{71}$. Finkelstein passa du RWR à l'American Russian Institute pour apporter son aide. Soutenu par Moscou et par des communistes

66 Dossier de Finley au FBI.

67 Information tirée de NAIDEN, «Finley's war years".

68 J'ai appris cela sur Mikhoels grâce à Alain Schnapp, qui l'a appris de Finley (communication personnelle, avril 2013).

69 Naiden : estimation de Levine donnée à une réunion du Jewish Council à Chicago, 30 octobre 1946 (Agence télégraphique juive, même date).

70 Naiden : matériaux anonymes et sans date, certains soviétiques, datant apparemment de 1944.

71 Dossier de Finley au FBI ; archives du National Council of American-Soviet Friendship (Tamiment Library, université de New York). 
américains de premier plan, directement contrôlé par le Parti communiste américain, l'ARI était une entreprise de relations publiques dynamique avec un personnel intéressant ${ }^{72}$.

La campagne d'adhésion échoua, ce qui ne saurait surprendre : le discours de Churchill sur le rideau de fer (5 mars 1946) avait contribué à changer le ton des relations internationales. Au bout de quelques mois, l'institut était dans le rouge à la banque et se déclara en faillite, ce qui rendit furieux les commanditaires qui reprochèrent à Finley d'être un « démolisseur ». Il démissionna de son poste ${ }^{73}$.

\section{La crise du printemps 1947}

Au printemps 1947, des sources documentaires auparavant clairsemées devinrent soudain visibles, et elles font penser à une évolution et une crise dans les rapports de Finley avec le Parti communiste : « Le 28 mars 1947 Finley démissionna de l'American Russian Institute. À partir de cette date nous n'avons aucune preuve de lien avec un groupe quelconque d'obédience communiste ou soviétique ${ }^{74}$."

« 30 mars 1947 : Finley demande à son vieil ami Benjamin Nelson, spécialiste de Weber, de lui rendre certains livres. P. S. À propos, depuis décembre dernier il s'appelle Finley $^{75}$."

"Avril 1947 : Finley écrit à son tuteur Westermann, qui décourage fortement un retour aux études universitaires. Finley propose qu'ils déjeunent ensemble. Il est réadmis à Columbia ${ }^{76}$."

72 On relève parmi les principaux responsables le nom de Harriet Moore (Bryn Mawr, promotion de 1932). Avant de partir pour l'URSS au milieu des années 1940, l'agent de Moscou Vladimir Kasakevich rapporta que Moore était pro-soviétique mais qu'elle « avait de la bouillie - kasha - dans le cerveau " (communication de Svetlana Chervonnaya, d'après la vOKS, septembre 2012). À en juger par les travaux de MoOre, p. ex. Soviet Far Eastern Policy 1931-45, Princeton, 1945, il avait tort. Kasakevich ramena sa femme, Emily Grace Kasakevich (Bryn Mawr, promotion de 1933, Ph.D. d'études classiques, Yale) à Moscou, où elle passa sa vie à écrire sur Démosthène. Elle correspondit régulièrement avec Finley.

73 Dossier de Finley au FBI.

74 Ibid.

75 Selon le dossier de Finley au FBI, le changement de nom eut lieu en octobre 1946.

76 La froideur de Westermann peut révéler qu’il se sentit « floué » par son étudiant. Six ans auparavant, Westermann avait assuré aux enquêteurs du Rapp-Coudert Committee (supra, note 66) que son brillant élève n'était pas membre du Parti : "Je suis convaincu que Moses I. Finley n'est certainement pas un communiste " (lettre à Ned H. Dearborn, 25 août 1941, Archives Boas) : Finley avait rapporté à Boas : "J'ai déjeuné avec Westermann [...] il est prêt à faire presque tout ce qu'il peut pour aider » (3 septembre 1941, Archives Boas). 
« 19 avril 1947 : Leo Lowenthal demande à Max Horkheimer une lettre de recommandation pour Finley."

" 20 avril 1947 : Finley écrit une nouvelle fois à Benjamin Nelson à propos de sa "décision qui mûrit lentement de retourner dans le monde d'où je viens". "

28 mai 1947 : Horkheimer fournit sa recommandation.

Un document inédit important mais ambigu est apparu à Moscou ; il m'a été envoyé par une historienne et archiviste de Moscou, Svetlana Chervonnaya. Dans son journal, A.A. Ermolaev, représentant à New York de la "Société soviétique pour les contacts culturels avec l'étranger ", dit que John Stuart, rédacteur à la revue New Masses, soutenue par le Parti, déclara le 20 mars : "Finley est un homme dévoué, membre du Parti communiste [...] mais il n'a pas défini sa place à l'American Russian Institute [...] et il ne comprend pas sa tâche ${ }^{77}$. " John Stuart était un homme bien informé (auteur avec Granville Hicks de John Reed: The Making of a Revolutionary, New York, 1936). C'est lui qui avait été choisi pour aller voir Granville Hicks lorsque ce dernier quitta le Parti en 1939. Ses dires sur l'appartenance au Parti contredisent les déclarations ultérieures de Finley à son doyen de Rutgers ${ }^{78}$. C'est le témoignage le plus " direct » dont nous disposons aujourd'hui sur cette question controversée, Finley était-il membre du Parti, mais il se situe à un moment de confusion, quelques semaines seulement avant que Finley quitte l'ARI pour n'avoir pas réussi à " comprendre sa tâche » et cesse tout contact visible avec le Parti. Et rien n'indique qu'il ait rejoint d'autres gens de gauche pour soutenir la campagne présidentielle de Henry Wallace en 1948.

En avril 1947, Leo Lowenthal loua la décision de Finley de retourner à l'université : «Dieu merci ! " Il fut peut-être soulagé aussi que son ami - un fils prodigue ? - se soit enfin éloigné des Russes.

77 Fond 5283, op. 22s, dossier 25, "Correspondance avec les États-Unis, dossier 23, vol. 2, 24 janvier-9 juin 1947 » de la voKs, la Société soviétique pour les contacts culturels avec les pays étrangers. Chervonnaya ajoute : "Ermolaev était alors le représentant de la VOKS à New York ; vraisemblablement du MGB, agissant à partir du consulat général soviétique. » Le MGB était le ministère de la Sûreté de l'État, une agence de renseignement.

78 Finley et d'autres connaissaient sûrement les difficultés juridiques qui accompagnaient l'adhésion au Parti après l'adoption de l'Alien Registration Act (le Smith Act) en juin 1940. L'historien de la Renaissance Charles Trinkhaus, qui connaissait Finley dans les années trente, et correspondit plus tard avec lui pendant des décennies, déclara devant une commission du Sénat qu'il avait été membre du Parti de 1937 à 1939 (Senate Subcommittee to Investigate the Administration of the Internal Security Act and Other International Security Laws, auditions du 4 juin 1953), mais il dit à l'Academic Freedom Committee du conseil d'administration du Sarah Lawrence College qu'il resta au Parti jusqu'en 1951. (Sarah Lawrence College Archives, note du président Harold Taylor, 20 octobre 1953). 


\section{Finley, Marx et les marxistes après 1947}

À partir de cette époque, Finley n'eut aucun contact visible avec des organisations marxistes ou russes. Il avait beaucoup d'amis à gauche mais ne fit pas ouvertement de politique partisane. Bien que ses travaux des années cinquante renvoient à un très large éventail d'influences intellectuelles - Weber, Nietzsche, Durkheim, Kant et d'autres -, il mentionne rarement Marx.

Cependant, les Archives Finley donnent une image quelque peu différente, grâce à une correspondance importante avec des savants du bloc soviétique, notamment Andreyev, Diakonoff et Emily Grace Kasakevich en URSS, Jan Pecirka à Prague, Detlev Lotze et Heinz Kreissig en Allemagne de l'Est. La correspondance de Lotze montre qu'il faut faire preuve de prudence dans la recherche : bien que vivant à Iéna, Lotze n'était ni membre du Parti ni marxiste, encore qu'il ait parfois utilisé des historiens marxistes comme Istvan Hahn ${ }^{79}$.

Dans une lettre à Pierre Vidal-Naquet, Finley dit avoir refusé une demande de John Saville et Ralph Milliband d'écrire sur " le marxisme et l'histoire ancienne " pour le Socialist Register, "à partir d'un réexamen de [George] Thomson » : "En principe je suis de plus en plus convaincu que ces articles "théoriques" vous font des ennemis et n'accomplissent pas grand-chose d'autre. Le seul espoir de laisser une marque sérieuse réside dans les études concrètes ${ }^{80}$. " Ce sont " les études concrètes ", pas la théorie ou l'historiographie marxistes, qui dominent sans aucun doute dans la correspondance avec Lotze, Kreissig et Pecirka : souvent, les discussions (comment interpréter Solon, que faire de Ruschenbush, la rémunération des charges) auraient aussi bien pu avoir lieu dans une université américaine. Lorsque les sujets " marxistes " sont abordés, ils ne sont pas très techniques : "Ce que vous dites sur la Hörigkeit, à savoir qu'elle exige des quantités de terres est, je dois l'avouer, un point nouveau pour moi. Il semble évident et il a des implications sur lesquelles je dois réfléchir encore ${ }^{81}$. " Il y a des discussions marxistes, comme lorsqu'Andrea Carandini soutenait que les quartiers d'habitation de Settefinestre étaient occupés par des esclaves, suggérant un «mode de production esclavagiste " qui était « en crise ». Finley n'était pas convaincu, ajoutant que le concept de "mode de production » était une abstraction dénuée de sens ${ }^{82}$. Bien entendu, dans les " modes de production », il y a le mode asiatique qui, après la mort de Staline en 1956

\section{Voir supra, note 7.}

80 Lettre à Pierre Vidal-Naquet, 12 septembre 1973, dans les Archives Vidal-Naquet, EHESS (consultées en 2006).

81 Finley à Lotze, 21 avril 1983, Archives Finley.

82 Lettre de Finley à Carandini, 2 juin 1980, dans les Archives Finley : "Bien que j’aie commencé à lire Marx avant que vous ne soyez né, je ne me permettrai pas de contester votre analyse approfondie, experte et détaillée. En outre, comme vous le savez, je crois, je ne comprends pas "mode de production"; ce n'est pas un concept que je peux utiliser ou refuser d'utiliser parce que pour moi il ne s'agit que de mots. Cela rend le débat difficile, c'est 
et la publication à l'Ouest des Grundrisse de Marx, acquit un poids considérable à l'Est dans les débats sur le changement historique. Avec sérieux, bienveillance et curiosité, Finley regardait Pecirka, Kreissig et Lotze en train de donner forme à la recherche sur le mode " asiatique ${ }^{83}$ ".

Dans son dernier essai, "Revolution in Antiquity ", Finley niait qu’il y ait eu de véritables révolutions dans l'Antiquité : "Nous nous retrouvons avec une espèce de tautologie : il n'y eut pas de transfert révolutionnaire du pouvoir vers une nouvelle classe (ou vers de nouvelles classes) parce qu'il n'y avait pas de nouvelles classes. » Fondamentalement, Finley nie que l'Antiquité ait connu la formation d'une classe ou un " changement véritable dans les fondements de l'État du point de vue des classes $^{84} »$.

Dans toutes ces discussions, Finley invoque sa paideia marxiste mais il l'évalue à l'aune d'un empirisme historique durement acquis. Si cette attitude a entraîné l'opprobre à l'Ouest, elle fut d'un grand secours dans ses efforts à l'Est, qui furent considérés comme une contribution importante au développement d'une histoire non dogmatique, notamment en Allemagne de l'Est. Les échanges de Finley avec ces chercheurs, certains extraordinairement doués, travaillant dans des conditions difficiles, sont un aspect important de son attitude réfléchie face au marxisme. Ces relations devront être étudiées, et ce sera avec profit.

Daniel TOMPKINS

Department of classics
Temple University
pericles@temple.edu

le moins qu'on puisse dire, et il ne servirait à rien que vous répondiez par une définition, même longue. J'ai lu suffisamment de définitions et je ne comprends toujours pas. "

83 Voir la correspondance avec Kreissig, Lotze et Pecirka dans les Archives Finley. Entre autres exemples de ce domaine de recherche très intéressant, on relève l'excellente étude de Pecirka à propos de la pensée soviétique sur le mode de production asiatique (J. PECIRKA, "Die Sowjetischen Diskussionen über das Verhältnis von Antike und Orient im Rahmen der Sklavenhaltformation ", Eirene 6 [1967], p. 141-174), et des essais de LoTZE ("Varianten der Produktionsweise ") et de Kreissig ("Fortschrifttliche Produktionsweise, revolutionäre Klasse. Gedanke zur Theorie der antiken Produktionsweise », JWG 2 [1967], p. 397-410). Je précise que cette recherche n'en est qu'à ses débuts.

84 "Revolution in Antiquity ", in R. Porter \& M. Teich (éd.), Revolution in History, Cambridge, 1986, p. 54, 56. 